\title{
ON ADDITION CHAINS
}

\section{ALFRED BRAUER}

We consider a set $a_{0}=1<a_{1}<a_{2}<\cdots<a_{r}=n$ of integers such that every element $a_{\rho}$ can be written as sum $a_{\sigma}+a_{\tau}$ of preceding elements of the set. Such sets of integers have been called "addition chains (Additionsketten) for $n$ " by A. Scholz. $\dagger$ For example, for $n=666$,

$$
1,2,4,8,16,24,40,80,160,320,640,664,666
$$

forms an addition chain with $r=12$; the same holds for

$$
1,2,3,6,9,18,27,54,81,162,324,648,666 .
$$

In any case, we must have $a_{1}=2$ and $a_{2}=3$ or 4 .

By the length $l=l(n)$ of $n$, Scholz understands the smallest $l$ for which there exists an addition chain $a_{0}, a_{1}, \cdots, a_{l}=n$.

The following question leads to addition chains: The least positive residue of $c^{n}(\bmod m)(c, m, n$ given integers) is to be formed using the smallest possible number of multiplications. Then $l(n)$ multiplications will always suffice.

A. Scholz published the following inequalities for $l(n)$ in the form of problems:

$$
\begin{gathered}
m+1 \leqq l(n) \leqq 2 m \text { for } 2^{m}+1 \leqq n \leqq 2^{m+1}, \quad m \geqq 1, \\
l(a b) \leqq l(a)+l(b) .
\end{gathered}
$$

In (1), we have $l(n)<2 m$ whenever $m>2$; moreover,

$$
l\left(2^{m+1}-1\right) \leqq m+l(m+1) .
$$

In connection with (3), Scholz surmises that (1) can be improved generally. He further raises the question of whether or not the inequality

$$
1 \leqq \limsup _{n \rightarrow \infty} \frac{\log 2}{\log n} l(n) \leqq 2,
$$

which easily follows from (1), can be improved.

It is easy to prove the formulas (1) and (2). I cannot decide whether (3) is always true. In the following, I will show that

$$
l\left(2^{m+1}-1\right) \leqq m+l^{*}(m+1),
$$

$\dagger$ Jahresbericht der deutschen Mathematiker-Vereinigung, class II, vol. 47 (1937), p. 41. 
where $l^{*}(m+1)$ is the minimal length, not of all, but only of certain addition chains. Further, I will prove by elementary methods that for sufficiently large $n$

$$
l(n)<\frac{\log n}{\log 2}\left\{1+\frac{1}{\log \log n}+\frac{2 \log 2}{(\log n)^{1-\log 2}}\right\} .
$$

This is better than (1). It entails the following relation

$$
\lim _{n \rightarrow \infty} \frac{\log 2}{\log n} l(n)=1,
$$

which, of course, is better than (4).

Let $a_{0}, a_{1}, \cdots, a_{l}=n$ be an addition chain for $n, 2^{m}+1 \leqq n \leqq 2^{m+1}$. Then $a_{\lambda} \leqq 2 a_{\lambda-1},(\lambda=1,2, \cdots, l)$. Since $a_{1}=2$, we have $a_{l} \leqq 2^{l}, n \leqq 2^{l}$, $2^{m}+1 \leqq 2^{l}, m_{\iota}+1 \leqq l$. This proves the first half of (1).

To prove the second part of $(1), l(n) \leqq 2 m$, suppose first that $2^{m}+1 \leqq n<2^{m+1}$. We write $n$ as a binary number

$$
n=2^{\nu_{1}}+2^{\nu_{2}}+\cdots+2^{\nu_{k}}, \quad \nu_{1}<\nu_{2}<\cdots<\nu_{k} .
$$

We have here at most $m+1$ terms, $k \leqq m+1$, and $\nu_{k}=m$. We begin the addition chain with $a_{0}=1, a_{1}=2, a_{2}=4, \cdots, a_{m}=2^{m}$, and take then

$$
\begin{aligned}
a_{m+1} & =2^{m}+2^{\nu_{1}}, a_{m+2}=2^{m}+2^{\nu_{1}}+2^{\nu_{2}}, \cdots, \\
a_{m+k-1} & =2^{m}+2^{\nu_{1}}+\cdots+2^{\nu_{k-1}}=n .
\end{aligned}
$$

This actually is an addition chain, and we see that $l(n) \leqq m+k-1$ $\leqq 2 m$. The equality $l(n)=2 m$ is possible only if $k=m+1$,

$$
n=1+2+2^{2}+\cdots+2^{m}=2^{m+1}-1 \text {. }
$$

This case will be discussed in the last paragraph of this page.

For $n=2^{m+1}$, we form the addition chain

$$
1,2,2^{2}, \cdots, 2^{m+1} \text {. }
$$

Here $l=m+1$, hence $l\left(2^{m+1}\right)=m+1 \leqq 2 m$.

Let $1, a_{1}, a_{2}, \cdots, a_{r}=a$ be an addition chain for $a$ with $r=l(a)$, and let $1, b_{1}, b_{2}, \cdots, b_{s}$ be one for $b$ with $s=l(b)$. Then

$$
1, a_{1}, \cdots, a_{r}, a_{r} b_{1}, a_{r} b_{2}, \cdots, a_{r} b_{s}
$$

forms an addition chain for $a_{r} b_{s}=a b$, since $b_{\rho}=b_{\sigma}+b_{\tau}$ implies $a_{r} b_{\rho}$ $=a_{r} b_{\sigma}+a_{r} b_{\tau}$. The number of terms after 1 in this chain is $r+s$; hence $l(a b) \leqq r+s=l(a)+l(b)$. This proves (2).

By a special addition chain for the number $n$ we mean an addition chain for which, for all $\rho$, and for some $\sigma$ 


$$
a_{\rho}=a_{\rho-1}+a_{\sigma}, \quad 0 \leqq \sigma \leqq \rho-1 \leqq l-1,
$$

holds. Let $l^{*}(n)$ be the minimal length of all special addition chains for $n$. Then $l(n) \leqq l^{*}(n)$. The chains used in the proof of the second part of (1) are special chains. Hence, it follows from this proof that $l^{*}(n) \leqq 2 m$. The equality sign is here impossible except for $n=2^{m+1}-1$. In order to prove that $l(n)<2 m$ whenever $m>2$, it suffices to show that

$$
l\left(2^{m+1}-1\right) \leqq l^{*}\left(2^{m+1}-1\right) \leqq m+l^{*}(m+1),
$$

for $1,2,4,5,6,7, \cdots, m+1$ is a special chain of length $m-1$ for $m+1$, so $l^{*}(m+1) \leqq m-1$. Let

$$
1=a_{0}, a_{1}, \cdots, a_{k}=m+1
$$

be a minimal special addition chain for $m+1, k=l^{*}(m+1)$. We form

$$
2^{a_{0}}-1=1,2^{a_{1}}-1=3,2^{a_{2}}-1, \cdots, 2^{a_{k}}-1
$$

and multiply $2^{a \kappa}-1$ successively $a_{\kappa+1}-a_{\kappa}$ times by $2,(\kappa=0,1,2, \cdots$, $k-1)$. We then obtain

$$
\left(a_{1}-a_{0}\right)+\left(a_{2}-a_{1}\right)+\cdots+\left(a_{k}-a_{k-1}\right)=m
$$

further numbers. We thus obtain the integers

$$
\begin{aligned}
& 1,2,2^{a_{1}}-1,2\left(2^{a_{1}}-1\right), 2^{2}\left(2^{a_{1}}-1\right), \cdots, 2^{a_{2}-a_{1}}\left(2^{a_{1}}-1\right), 2^{a_{2}}-1, \\
& 2\left(2^{a_{2}}-1\right), \cdots, 2^{a_{3}-a_{2}}\left(2^{a_{2}}-1\right), \cdots, 2^{a_{k}-a_{k-1}}\left(2^{a_{k-1}}-1\right), 2^{a_{k}}-1 .
\end{aligned}
$$

We state that these numbers form a special chain for $2^{a_{k}}-1=2^{m+1}-1$. This will be proved if we show that

$$
2^{a_{\kappa}}-1-2^{a_{\kappa}-a_{\kappa-1}}\left(2^{a_{\kappa-1}}-1\right)=2^{a_{\kappa}-a_{\kappa-1}}-1
$$

is an element of (8) for $\kappa=2,3, \cdots, k$. But this is true, since (7), as a special addition chain, contains $a_{k}-a_{k-1}$. The length of the chain (8) is $k+m=l^{*}(m+1)+m$, and this proves (6).

We show now that A. Scholz' conjecture, that (1) and (4) can be improved, is actually true. We prove the following theorem:

THEOREM. If $r$ is any positive and $s$ any not negative integer, then

$$
l(n) \leqq(r+1) s+2^{r}-2 \text { for } 2^{r s} \leqq n<2^{r(s+1)} .
$$

Proof. When $r=1$, this follows from (1); we therefore take $r>1$ and fixed. I state now that we can form an addition chain for $n$ which contains at most $(r+1) s+2^{r}-2$ terms, and which begins with the terms $a_{0}=1, a_{1}=2, a_{2}=3, \cdots, a_{2^{r}-2}=2^{r}-1$. For $s=0$ this is true because the integers $1,2, \cdots, 2^{r}-1$ form an addition chain for every 
$n<2^{r}$ (the integers $n+1, n+2, \cdots, 2^{r}-1$ may be included in the chain). Assume now that the assertion is not true and take $s$ to be the smallest value for which the statement does not hold for $n$ with $2^{r s} \leqq n<2^{r(s+1)}$. We divide $n$ by $2^{r}$ :

$$
n=a \cdot 2^{r}+b, \quad 0 \leqq b<2^{r} .
$$

Then $2^{r(s-1)} \leqq a<2^{r s}$, and since our statement is supposed to be true for $s-1$ instead of $s$, there exists an addition chain $a_{0}, a_{1}, a_{2}, \cdots$, $a_{\alpha-1}, a_{\alpha}=a$ for $a$ which has at most $(r+1)(s-1)+2^{r}-2$ terms, and which starts with $1,2, \cdots, 2^{r}-1$. Because of $(10)$, this chain contains $b$ for $b>0$. Then $a_{0}, a_{1}, \cdots, a_{\alpha-1}, a, 2 a, 2^{2} a, \cdots, 2^{r} a, 2^{r} a+b$ is an addition chain for $n$ which contains the first $2^{r}-1$ integers. The length equals at most

$$
(r+1)(s-1)+2^{r}-2+r+1=(r+1) s+2^{r}-2 .
$$

This gives the desired contradiction; therefore the statement holds for all values of $s$. The proof yields an easy method for constructing the addition chains.

From relation (9) it follows that $s \leqq(\log n) / r \cdot \log 2$; hence $l(n)$ $\leqq(r+1)(\log n) /(r \log 2)+2^{r}-2$. If now $2^{m} \leqq n<2^{m+1}$, this yields

$$
l(n) \leqq \min _{r=1,2, \cdots, m}\left\{\left(1+\frac{1}{r}\right) \frac{\log n}{\log 2}+2^{r}-2\right\} .
$$

For instance, if we set $r=[\log \log n]+1$ for $n \geqq 3$, then (11) gives

$$
\begin{aligned}
l(n) & <\left(1+\frac{1}{\log \log n}\right) \frac{\log n}{\log 2}+2^{\log \log n+1} \\
& =\left(1+\frac{1}{\log \log n}\right) \frac{\log n}{\log 2}+2 e^{\log \log n \cdot \log 2} \\
& =\frac{\log n}{\log 2}\left(1+\frac{1}{\log \log n}\right)+2(\log n)^{\log 2} \\
l(n) & <\frac{\log n}{\log 2}\left\{1+\frac{1}{\log \log n}+\frac{2 \log 2}{(\log n)^{1-\log 2}}\right\} .
\end{aligned}
$$

This inequality can easily be improved since the expression between the braces in (11) takes on its minimum for $r^{2} \cdot 2^{r}=(\log n) /(\log 2)^{2}$.

On the other hand, it follows from (1) that $l(n) \geqq m>(\log n) /(\log 2)$ -1 . This, in connection with (12) yields $\lim _{n \rightarrow \infty} l(n)(\log 2) /(\log n)=1$.

The Institute for Advanced Study 